\title{
ELEMENTS IN THE COSMOS: ORIGIN OF THE LIGHT ELEMENTS.
}

\author{
Hubert Reeves \\ Service d'Astrophysique \\ C.E.N.S Saclay \\ Institut d'Astrophysique de Paris .
}

ABSTRACT. In the first part of this paper, a review is given of the situation of the Big Bang nucleosynthesis of the nuclides $\mathrm{D},{ }^{3} \mathrm{He},{ }^{4} \mathrm{He}$ and ${ }^{7} \mathrm{Li}$, taking into account the latest experimental data (number of neutrino species, lifetime of the neutron) and theoretical developments (quark-hadron phase transition). In the second part. I review the process of Galactic Cosmic Ray formation of lithium, beryllium and boron throughout the life of the galaxy, taking advantage of recent measurements of $\mathrm{Be}$ and $\mathrm{Li}$ in iron deficient stars,

\section{1- Cosmological nucleosynthesis.}

The game of Big Bang Nucleosynthesis (BBN) is to compute the abundances of light nuclides $X_{i}$ (the "yields") generated around $T=10^{9} \mathrm{~K}$ and to compare them with the observed abundances. To reach this goal we must face two different tasks : 1) to follow the physics of the Big Bang forward in time until $10^{9} \mathrm{~K}$, in order to obtain a set of physical parameters from which the $\mathrm{X}_{\mathrm{i}}$ can be computed, 2) to extrapolate the chemical abundances from the observed data, all the way back to $10^{9} \mathrm{~K}$. Then, try to match the calculated and extrapolated abundances $X_{i}$ at the "interface" of BBN, around $10^{9} \mathrm{~K}$.

I discuss first the computations of the yields. In the recent years, we have had important progresses but also some unforeseen and unpleasant complications.

Two new important laboratory experiments have resulted in a decrease in the number of "effective" parameters needed for the computations. From the recent LEP results, (CERN collaboration 1990) we have obtained the number of neutrino species ( $3.2 \pm 0.2)$. This number is of importance in relation with the cosmic energy density and hence with the rate of expansion of the universe. This rate, in turn, fixes the value of the decoupling temperature of the weak interaction. The neutron density at the decoupling temperature fixes the calculated abundance of helium-4. In the early computation of BBN, this number was left as a "parameter " to be fitted by comparison with the observed abundances. For years it had been foreseen that the number of neutrino species had to be close to the presently measured value of three. (Yang et al 1984) It is worthwhile recalling here than in BBN computations, "fractional" numbers of relativistic species are not necessarily meaningless since they could correspond to species which would have decoupled at earlier times.

From the Grenoble cold neutron laboratory (Mampe et al 1989) we have an improved value of the neutron lifetime. The value : lifetime of $890 \pm 4 \mathrm{sec}$ (corresponding to an half-life of $10.3 \pm 0.06$ minutes) is accurate enough that one does not have to include the influence of its 
uncertainty on the yields. This fact effectively reduces again the number of parameters of BBN.

The only remaining parameter of $\mathrm{BBN}$ is the density of baryonic matter. In the past years, it was generally assumed that this density was spatially homogeneous throughout the universe at the moment of BBN. In this case (usually called the "standard BBN), the computed yields are found to match reasonably well the extrapolated observations of light nuclides in the density range from 2 to $4 \times 10^{-31} \mathrm{gr} / \mathrm{cm}^{3}$ (or $\eta=3$ to $6 \times 10^{-10}$ : the ratio of the number of nucleons to the number of photons) (Yang et al 1984) (Pagel 1989) (Beaudet and Reeves 1984).

Over the recent years, we have progressively come to realise that a realistic computation of BBN should take into account the possible effects of the quark-hadron phase transition around $200 \mathrm{MeV}$ (Witten 1984) (Satz 1985). In view of this developpements, it seems that we should no more use the word "standard" for the models assuming homogeneity in baryonic density nor should we use the words "non standard Big-Bang" to describe models taking into account the reality of this phase transition.

One major question here is the order of the quark-hadron phase transition. If it is of second order, it creates no density inhomogeneities and we can simply use the homogeneous density model. If it is of first order, baryonic density inhomogeneities are likely to have been created during the transition, when the universe was approximately twenty microseconds (at the conventional cosmic clock). The size of the horizon was then ten kilometres and the mass within the horizon around $10^{26}$ grams. This number gives an upper limit to the size of the density inhomogeneities possibly created at this instant.

From this time until about one second, the weak interactions were fast enough to insure a uniform neutron to proton ratio (given by the Boltzman factor : $\left(n / p=\exp \left(M_{n}-M_{p}\right) / k T\right)$ ) throughout the expanding inhomogeneities, despite the fact that the neutrons diffused away from the density condensations while the protons were kept inside by their electromagnetic interactions with the photon gas. Around one second, the weak interactions became too slow to insure this weak interaction equilibrium any more. The neutrons diffused away faster than the protons and $\mathrm{n} / \mathrm{p}$ inhomogeneities were created. Around $0.1 \mathrm{MeV}$ (one hundred seconds) primordial nucleosynthesis began, first in the high density regions. The capture of neutrons to form deuterons decreased the neutron density and generated neutron back-diffusion from the low density to the high density regions, The computations have to take into account this phenomena, in a dynamical way, in order to obtain realistic yields.

This crucial question of the order of the transition should be answered by high energy collisions of heavy nuclei. Experiments have already started at CERN. The preliminary tests, based on the number of J/ $\Psi$ particles emitted (Satz 1987) (Potvin 1989), indicates that the state of quark-gluon plasma has been reached during the collisions. However the correct interpretations of the results is a matter of controversy. It is expected that definite conclusions are still a long way in the future.

The question can also be studied through QCD calculations on lattices along a method initiated by Wilson and Polyakoff. The results already published in the past years (Akura 1990) are not free of difficulties and still involve a a number of simplifying assumptions (Ukawa 1989). Year after years, the situation is improving but we are still far from having definite answers. The last "news from the lattice" (Fukujita et al 1990) favors a first order phase transition. This result however is based on so-called "pure gauge fields", meaning that only the effects of the gluons have been taken into account but not the effects of the quarks themselves. Pending better QCD results and forthcoming experimental data, I will assume that the transition is indeed first order. In order to cover all uncertainties, the effects of the phase transition are taken into account by introducing a new set of parameters. As far as the yields are concemed, we have to face spatial variations of the baryonic density: $\rho_{b}$ becomes $\rho_{b}(r)$. This space dependance is usually dealt with by the introduction of three new parameters : $d$, the mean distance between the condensation peaks, $R$, the density contrast between the maxima and the minima, and $f_{v}$ a measure of the "clumpiness" of the medium.

The value of the mean distance $d$ depends on the surface energy of the "bubbles" of 
hadrons nucleating in the sea of quarks during the transition. If this surface density ( $s$ ) is high, overcooling will be extended, only large bubbles will form and there will be few of them : thus the value of $\mathrm{d}$ will also be large. If $\mathrm{s}$ is low, many small bubbles will form early and $d$ will be low. We expect the value of $s$ to be given by QCD calculation on a lattice (Potvin 1990). In our present ignorance of the value of $s$ we have to face the possibility that $\mathrm{d}$ could extend all the way from zero to the value of the horizon at Q-H phase transition (ten kilometers).

Qualitatively the situation is the following. In the upper part of the range $(1<\mathrm{d}<10$ $\mathrm{km}$ at Q-H transition) the distances between the bubbles are too large for appreciable neutron diffusion before and during $B B N$. In consequence we have density inhomogeneities but no $\mathrm{p} / \mathrm{n}$ inhomogeneities. The yields are obtained by summing and averaging the results of constant density yields over the various density regions. At the other extreme, if $\mathrm{d}$ is less than $0.1 \mathrm{~m}$, both the neutrons and the protons diffuse and the density is homogenize before BBN. The intermediate case $(1 \mathrm{~km}>\mathrm{d}>0.1 \mathrm{~m})$ is the crucial one where the neutrons diffuse effectively but not the protons.

The contrast $R$ depends, in part, upon the value of the critical temperature $T_{c}$ which is expected to be between 100 and $250 \mathrm{MeV}$. In the lower part of the range $\left(\mathrm{T}_{\mathrm{c}}<150\right.$ $\mathrm{Mev}$ ), this computed contrast $\mathrm{R}$ tends to be large (several tens). It decreases gradually toward the upper end of the range, as simple phase space argument would predict. The value of $T_{c}$ should come out from QCD calculations. There are already some indications, from perturbative approaches, that it should lie in the upper part of the range (Gasser and Leutwyler, 1987, 1988).

Finally, the value of $f_{v}$ depends in a complicated way on the hydrodynamics of the hadron bubble growth. The problem is that QCD calculations on lattices can only deal with statistical equilibrium situations; they are unable to treat dynamical processes (in these calculational techniques, the parameter "time" is replaced by the "temperature"). The problem is not with the universal expansion (too slow to influence the course of events) but with the rate of growth of the bubbles.

Simplified models have been made of these processes, based on the hypothesis of weakly interacting particles (Miller and Pantano 1989) (Fuller et al 1988). In these models the pressure and the energy density are given by the product of the number of particle species (with appropriate multiplicity factors) and the fourth power of the temperature. The transition then corresponds to an abrupt change of this demographic factor (from 37 for the quark-gluon plasma to 3 in the hadronic phase). However there are reasons, based on QCD calculations to doubt the validity of the weak interaction hypothesis close to the critical temperature.

In view of all these uncertainties in the values of our three parameters, the standard procedure has been to cover the whole parameter space, computing with appropriate averaging the corresponding yields. These calculations, including fine zoning and neutron back-diffusion, have been made by several groups (Mathews et al 1988, Reeves et al 1990, Terasawa and Sato 1989, Kurki-Suonio et al 1989 Applegate et al 1988). The results are in general agreement.

With all these numbers, one is in position to evaluate the range of mean baryonic density compatible with the observations. More specifically we ask the question : in what fraction of the parameter space of $d, R, f_{v}$ do we find appropriate yields? This question necessarily introduces an element of subjectivity in the decision of the minimum fraction acceptable. However the expected chaotic hydrodynamic processes accompanying the bubbling and percolation probably imply a rather wide dispersion of these effective parameters around their mean values. Since the yields are often strong function of these parameters, this dispersion would seem to argue against any scenario 
based on a very small fraction of the parameter space (narrow choice of parameters).

I will discuss the situation with respect to the baryonic density range (Reeves 1990).The lower range (from 1 to $2 \times 10^{-31} \mathrm{~g} / \mathrm{cm} 3$ ) would require heavy $\mathrm{D}$ astration initial $\left(\mathrm{D} / \mathrm{H}>3 \times 10^{-4}\right.$ ), which is made unlikely by the present upper limit value of $\left((\mathrm{D}+3 \mathrm{He}) / \mathrm{H}<10^{-4}\right)$. In the range from 2 to $10 \times 10^{-31}$, we find acceptable yields in a large part of the parameter space, with a tendancy toward larger $R$ values $(R>10)$ as we move toward higher densities. In the range from 10 to $20 \times 10^{-31}$, the acceptable area in the space parameter shrinks rapidly. Values of $R$ $\gg 10$ and narrow ranges of $f_{v}$ are required in order to fit the data. Above $20 \times 10^{-31}$ the required values of $\mathbf{R}$ reach several thousands. Even at that, the He yields are always larger than 0.25 and the $\mathrm{Li} / \mathrm{H}$ always larger than $10^{-9}$. My subjective feeling is to select a compatible mean baryonic density range between 2 and $10 \times 10^{-31},\left(3 \times 10^{-10}<\eta<15 \times 10^{-10}\right)$. Rather similar bounds on the mean baryonic density acceptable have been obtained by Kurki-Suonio et al (1990) and by Pagel (1990).

As the QCD calculations on lattices proceeds, quantitative evaluations of the parameters will improve progressively, and the range of acceptable baryonic density will undoubtely narrow down.

Some authors (Malaney and Fowler 1989, Mathews et al 1989, Applegate, Hogan and Scherrer 1987) have considered the possibility of $\Omega_{b}=1$, (corresponding to $\rho_{b}=10^{-29}$ for $H_{0}=75 \mathrm{~km} / \mathrm{sec} / \mathrm{Megaparsec}$ ). Beside requiring unrealistically large values of the primordial $\mathrm{Li}$ abundance, this scenario corresponds to very narrow choices of the parameters (which also appears somewhat unrealistic). These authors have studied the formation of the light elements $\mathrm{Li} \mathrm{Be} \mathrm{B}$ and the r-elements in this scenario. It already appears unlikely that, even with these extreme assumptions, they could produce interesting amounts of these elements. Observational data on old stars will be of prime importance to decide (Rebolo 1990).

\section{2- Physical processes in the early galaxy}

In addition to their interest as cosmological observables, the nuclear species lighter than carbon $(\mathrm{Z}<6)$ are potentially rich indicators of the physical conditions accompanying the formation of our galaxy.

In this respect the recent observations of $\mathrm{Be}$ and $\mathrm{Li}$ in old PopII stars are particularly worth discussing here (Ryan et al 1990) (Rebolo et al 1988). In (figure 1) are shown the abundances of $\mathrm{Be}$ as a function of metallicity $(\mathrm{Fe} / \mathrm{H})$. At $(\mathrm{Fe} / \mathrm{H})=-1$ (one tenth of the solar iron abundance) the Be abundance is approximately one tenth of its Pop I abundance. At lower metallicity, no Be has yet been observed but the upper limit fall down by another order of magnitude.

This drop in the Be abundance is not unexpected since, according to the galactic cosmic ray (GCR) origin of the light elements Li Be B, (Meneguzzi, Audouze, Reeves 1970, Reeves 1974, Boesgaard and Steigman 1985 Arnould and Forestini 1989) these atoms are produced by time accumulated effect of the bombardment of fast particles on heavier atomic targets of the galactic gas and dust, mostly carbon and oxygen.

From the energy spectrum of galactic cosmic protons (at solar minimum activity), presented in (figure 2), it appears clearly that most of the bombarding particles have kinetic energies between $200 \mathrm{MeV}$ and $10 \mathrm{GeV}$. The nuclear excitation functions for the production of light nuclides by 

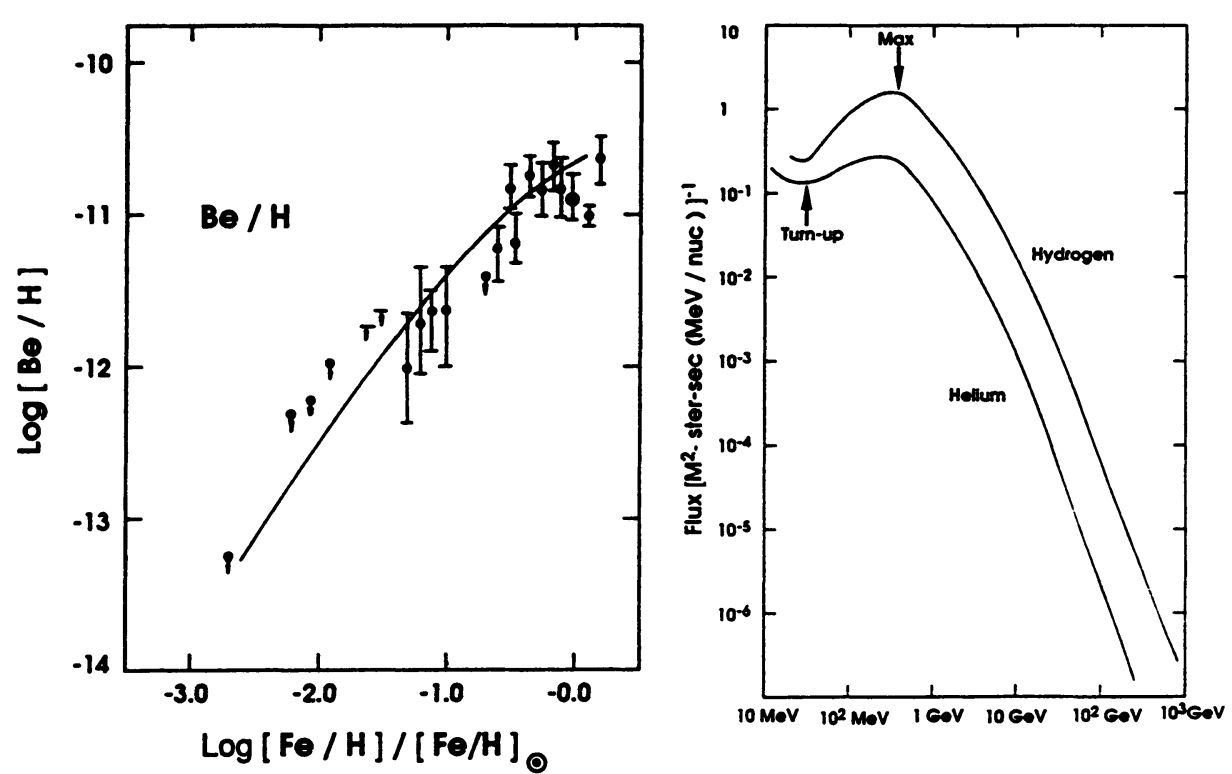

Figure 1: Stellar abundances of $\mathrm{Be}$ as a function of metallicity $(\mathrm{Fe} / \mathrm{H})$. At one tenth of the solar iron abundance, the beryllium abundance is down by a factor of ten.

Figure 2: Energy spectrum of galactic cosmic rays protons and alphas at solar minimum. Most of the particles are in range from $300 \mathrm{MeV}$ to $3 \mathrm{GeV}$

the collision of fast protons on oxygen targets (figure 3) and on carbon targets (figure 4) are almost constant in this energy interval. Thus the rate of formation of these nuclides is essentially given by the product of the cross-sections at high energies with the flux of incoming protons.

For the observations of Be in PopII stars, two factors are expected to result in abundances lower than in PopI stars : shorter time exposition and lesser target abundances. Each of these factors should decrease the PopII abundance by approximately one order of magnitude with respect to the PopI abundance. In this respect, the Be abundance at $(\mathrm{Fe} / \mathrm{H})=-1$ (one tenth of the PopI abundance) is surprisingly high.

More realistically we should not expect simple models of irradiation (as in Reeves and Meyer 1978) to be valid in this context. We enter here in the still largely mysterious period of galactic formation, with its possible relation to galactic environment and collisions (the galaxies were much closer). The ordinary leaky box models, with particle escape range governed by the scale of magnetic inhomogeneities, are probably to be considered anew (Casse 1990). The Be data should be used to give information on the general scenarios of galactic birth and youth.

The contribution of G.C.R. to the abundances of the other light nuclides $\left({ }^{6} \mathrm{Li},{ }^{7} \mathrm{Li}{ }^{10} \mathrm{~B}\right.$, ${ }^{11} \mathrm{~B}$ ) is essentially given by the ratio of the spallation cross-sections on $\mathrm{C}$ and $\mathrm{O}$ (the special case of the alpha + alpha reactions will be considered later). In (figure 5) the corresponding abundance curves are shown. While these curves give a reasonable fit to the abundance of ${ }^{6} \mathrm{~L}$ and $B$ in the solar system (with a discrepancy of approximately a factor of two for the boron isotopic ratio, see Meyer and Reeves for a discussion), the ${ }^{7} \mathbf{L i}$ abundance is strongly underestimated both in in the PopII and in the PopI region.

This brings us to a second major piece of information on the early galaxy: the measurement of lithium abundances in PopII stars . The data of several groups (Rebolo et al 1988), (Rebolo et al 1987), (Hobbs and Pilachowski 1988), (Hobbs and Duncan 1987), (Spite et al 1984), 


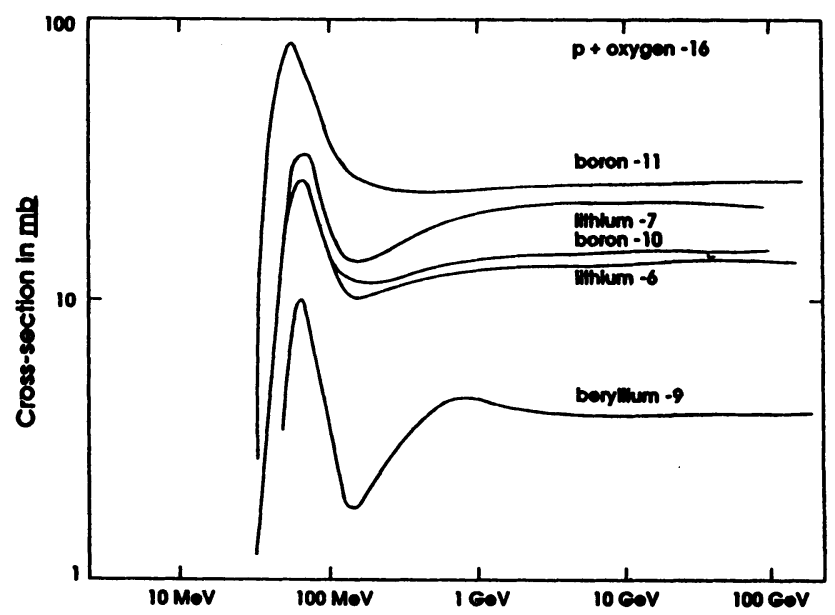

Figure 3: Formation cross-section of various nuclides by collisions of fast protons on oxygen. Oxygen is the most abundant target in space. The cross-sections are almost constant from $300 \mathrm{Mev}$ on up. The Be cross-section is down by one order of magnitude with respect to $\mathrm{Li}$ and $\mathrm{B}$ isotopes

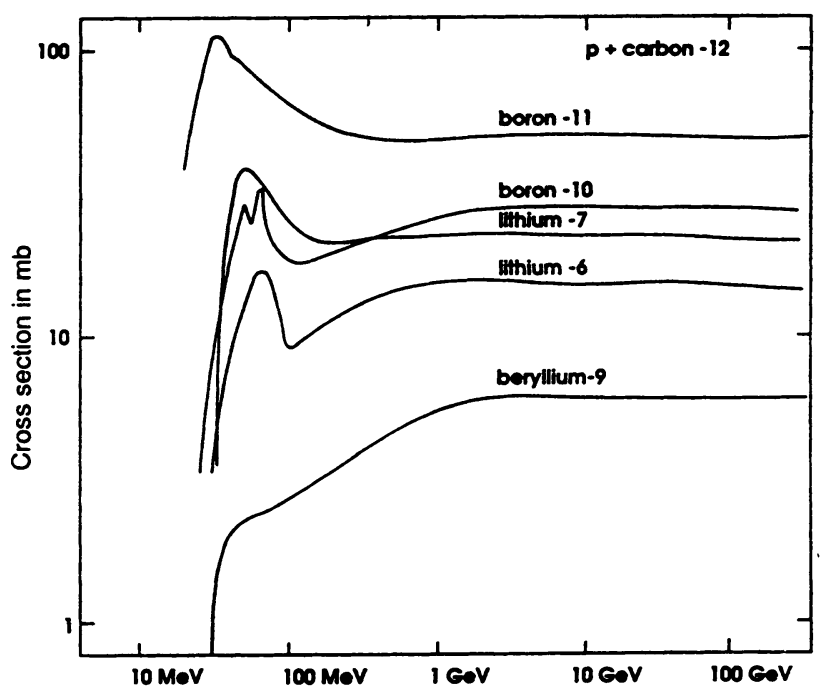

Figure 4: Formation cross-section of various nuclides by collisions of fast protons on carbon. The situation is quite similar to the case of oxygen. 


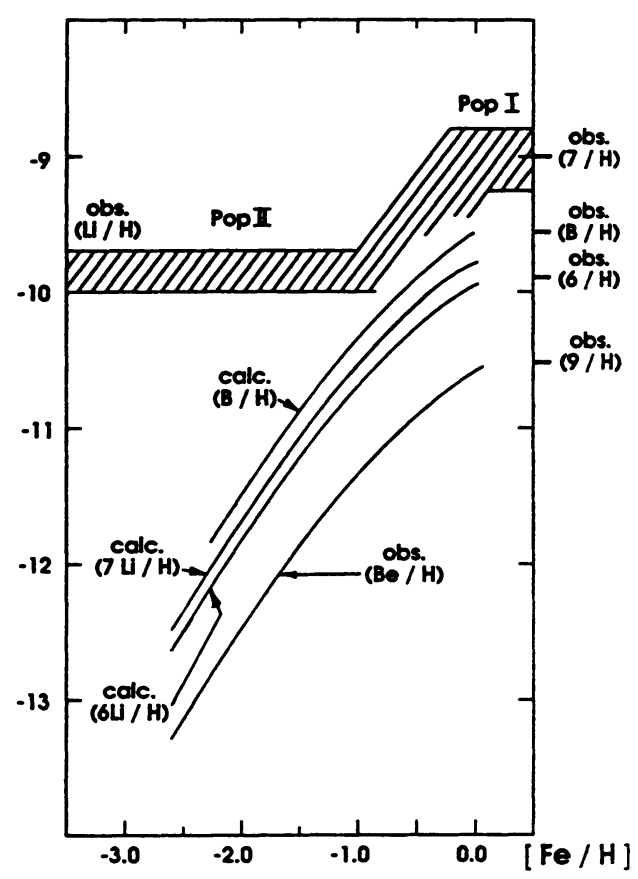

Figure 5: Abundance of the light nuclides ${ }^{6} \mathrm{Li},{ }^{7} \mathrm{Li}{ }^{9} \mathrm{Be}, \mathrm{B}$ generated by galactic cosmic rays on interstellar $\mathrm{C}$ and $\mathrm{O}$, as a function of galactic iron abundances. The curves are calculated from the Be observations taking into account the relative nuclear formation cross-sections on $\mathbf{C}$ and $\mathrm{O}$. The observed abundances at present time are shown on the scale at the left. Also shown are the lithium abundances, with their dispersions.

(Spite and Spite 1982), (Spite et al 1987), is presented in (Figure 6) as a function of metallicity. The most remarkable fact is the constancy of the $\mathrm{Li}$ abundance (dispersion of about a factor of two) for (Fe/H) less than -1). From there, several conclusions can be obtained : 1) this lithium component is not of stellar origin, 2) neither was it made by the bombardment of G.C.R on interstellar $\mathrm{C}$ and $\mathrm{O}$ (see fig 5); 3) one can obtain a lower limit to the production of the heavy elements $(\Delta Z)$ per unit mass $(\Delta M)$ of matter retumed to the interstellar gas after astration. If this ratio had been smaller than $0.2(\Delta \mathrm{Z} / \Delta \mathrm{M}<0.2)$ we would see a decrease of the $\mathrm{L} / \mathrm{H}$ ratio with increasing $(\mathrm{Fe} / \mathrm{H})$, due to increasing dilution of $\mathrm{Li}$ in $\mathrm{H}$.

\section{3- Pregalactic cosmic rays ?}

We consider the possibility that the PopII lithium could have been generated hypothetical pregalactic cosmic rays. The collisions of primordial helium nuclei could have generated interesting amounts of ${ }^{6} \mathrm{Li}$ and ${ }^{7} \mathrm{Li}$, together with $\mathrm{D}$ and ${ }^{3} \mathrm{He}$. The excitation function for the production of ${ }^{6} \mathrm{Li}$ and ${ }^{7} \mathrm{Li}$ by alpha+ alpha is shown in (figure 7) (from Read and Viola 1984). Except in the threshold energy region, the cross section for ${ }^{6} \mathrm{Li}$ production is an order of 
magnitude larger than the cross section for ${ }^{7} \mathrm{Li}$ production. This is as expected from phasespace arguments: the ${ }^{7} \mathrm{Li}$ corresponds generally to a three-body break-up, while the ${ }^{7} \mathrm{Li}$ corresponds to a two body break-up.

The ${ }^{6} \mathrm{Li}$ abundance at solar birth (essentially the only ${ }^{6} \mathrm{Li}$ observation at our disposal!) can be used to derive an upper limit to the pregalactic ${ }^{7} \mathrm{Li}$ contribution. Using the value $6_{\mathrm{Li} / \mathrm{H}}=$ $10^{-10}$ in the protosolar nebula, and taking into account the fact that ${ }^{7} \mathrm{Li}$ formation cross-sections are approximately ten times smaller than the ${ }^{6} \mathrm{Li}$ cross-sections, we obtain an upper limit of $10^{-11}$ for this contribution. This is only tenth of the PopII abundance.This argument is not completely tight since one could further assume that the hypothetical pregalactic cosmic rays have a mean energy around 10 to $20 \mathrm{Mev}$; close to the threshold energies of these reactions. At these energies the cross sections are quite similar. The

upper limit of the abundance of lithium thereby generated could then be comparable to the PopII abundances. However this extra assumptions make this scenario rather unlikely.

It is worth saying that, in all these cases, the calculated amount of $D$ and ${ }^{3} \mathrm{He}$ generated by this mechanism is always several orders of magnitude smaller than observed. We still need BBN to generate the observed abundance of D ( ${ }^{3} \mathrm{He}$ could be produced by stellar processes).
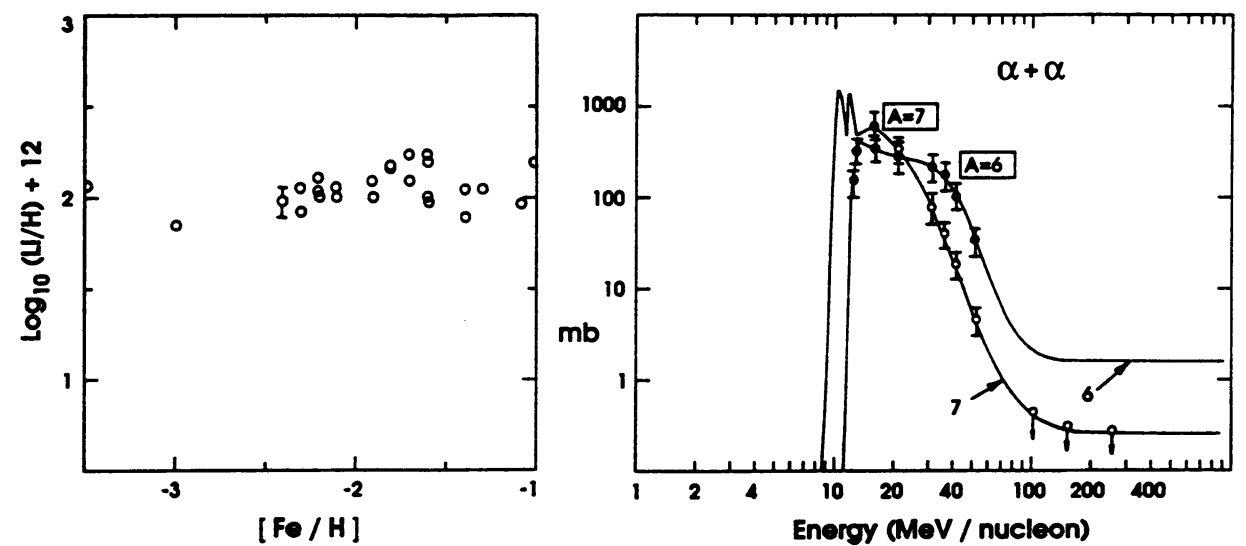

Figure 6: Stellar abundances of lithium as a function of metallicity for iron metallicity lessone tenth of solar.

Figure 7: Excitation functions for the production of ${ }^{6} \mathrm{Li}$ and ${ }^{7} \mathrm{Li}$ by alpha+ alpha collisions. 


\section{References}

Applegate, J. H., Hogan, C., Sherrer, R.J., 1987, Phys. Rev. D 35, 1151.

Applegate, J. H., Hogan, C., Sherrer, R.J., 1988, Astrophys. J. 329, 572.

Amould, M., Forestini, M., 1989 Research Report in Physics : Nuclear Astrophys., eds M. Lozano, M. L. Gallardo, J. M. Arias, p. 48, Springer, Berlin.

Beaudet, G., Reeves, H., 1984, Astron. Astrophys 134, 240.

Boesgaard, A.M., Steigman, G., 1985, Ann. Rev. Astr. Ap. 23, 319.

Boyd, R.N., Kajino, T., 1989, Astrophys. J. Letters 336, L55.

Cassé, M., work in progress, 1990.

Cayrel, R., Proceedings of the Alpbach Summer school 1988, CERN Collaboration ALEPH DELPHI, L3 OPAL MARK II, preprint, Geneva 1989.

Charbonneau, P., Michaud, G., 1988, Astrophys. J. 327, 809.

Deliyannis, C.P., Demarque, P., Kawaler, S.D., Krauss, L.M., Romanelli, P., 1987, Phys. Rev. Letters 62, 1583.

Deliyannis, C.P., Demarque, P., Kawaler, S.D., preprint Yale 1989, 1990, Astrophys. J., in press.

Fukujita et al, Nucl. Phys. 1990, Proc. Suppl.

Fuller, G.M., Mathews, G.J., Alcock, C.R., 1988, Phys. Rev. D37, 1380.

Gasser, J., Leutwyler, H., 1988, Phys. Lett. B. 8 3, 184.

Gasser, J., Leutwyler, H., 1987, Phys. Lett. B. 188, 477.

Hobbs, L.M., Pilachowski, C., 1988, Astrophys. J. Letters 326, L23.

Hobbs, L.M., Duncan, D.K, 1978, Astrophys. J. 317, 796.

Irbäck, A., Karsh, F., Peterson, B., Wyld, H.W., 1988, preprint, CERN TH 5130/88.

Kunth, D., Sargent, W., 1983, Astrophys. J. 273, 81.

Kurki-Suonio, H., Matzner, R.A., Centrella, J.M., Rothman, T., Wilson, J.R., 1988, Phys. Rev. D 38, 1091.

Kurki-Suonio, H., Matzner, R.A., Olive, K.A., Schramm, D.N., 1990, Astrophys. J., in press.

Malaney, R.A., Fowler, W.A, 1988, in Origin and Distribution of the Elements, ed. Mathews, G. J., World Scientific, Singapore.

Malaney, R.A., Fowler, W.A, 1989, Astrophys. J. 345, L5.

Mampe, W., Ageron, P., Bates, J., Pendebury,J., Steyerl, A., 1989, Phys. Rev. Lett. 62, 593 Proc. Grenoble Workshop on slow neutrons, Nuclear Instruments and Methods.

Matteucci, F., 1987 in Proc. ESO Workshop on Stellar Evolution and Dynamics of the outer halo of the Galaxy, eds. M. Azzopardi, F. Matteucci (Garching ESO), p.609

Mathews, G., Meyer, B., Alcock, C.R., Fuller, G.M., UCRL Preprint 102320.

Mathews, G., Fuller, G.M., Alcock, C.R., Kajino, 1988, preprint UCRL 98943.

Menneguzzi, M., Audouze, J., Reeves, H., 1971, Astron. Astrophys. 15, 337.

Michaud, G., Charbonneau, P., 1990, Space Science Rev., in press.

Miller, J.C., Pantano, O.,1989, preprint SISSA Trieste.

Pagel, B.E.J., Proceedings of the NATO Advanced Study Institute on Baryonic Dark Matter, Cambridge, July 1989, G. Gilmore, D. Lynden-Bell (eds).

Pagel, B.E.J., 1987 in $A$ unified view of the macro- and micro-cosmos, First International School on Astroparticle Physics, Erice (Sicily, Italy ) Ed: A. De Rujula, N.V. Nanopoulos, P.A. Shaver, World Scientific, Singapore.

Pagel, B.E.J., Simonson, E.A., 1989, to appear in Rev. Mex. Astr. Astrophys.

Pagel, B.E.J. 1989 in Evolutionnary Phenomena in Galaxies, eds J.E. Beckman, B.E.J. Pagel, Cambridge University Press.

Pinsonneault, M.H., Kawaler, S.D., Sofia, S., Demarque, P., 1989, Astrophys. J. 338, 424. 
Potvin, J., 1989, Can. J. Phys. 67, 1236.

Read, S.M., Viola, V.E., 1984, Atomic Data Nucl. Data Tables 31, 359.

Rebolo, R., Molaro, P., Abia, C., Beckman, J.E, 1988, Astron. Astrophys. 193, 193.

Rebolo, R., Molaro, P., Beckman, J., 1988, Astron. Astrophys. 192, 192.

Rebolo, R., Beckman, J., Molaro, P., 1987, Astron. Astrophys. 172, L17.

Rebolo, R., 1990, Proceedings of the Cambridge Conference on: Elements in the cosmos.

Reeves, H., 1974, Ann. Rev. Astron. Astrophys. 12, 437.

Reeves, H., Meyer, J.P.M., 1978, Astrophys. J. 226, 613.

Reeves, H., 1990, Physics Reports, in press.

Reeves, H., Richer, J., Sato, K., Terasawa, N., 1990, Astrophys. J. 355, 18.

Ryan, S.G., Bessell, M.S., Sutherland, R.S., Norris, J.E., 1990, Astrophys. J. Lett. 348, L57.

Satz ,H.,1985, Ann. Rev. Nucl. Sci. 35.

Satz, H.,1987, Proceedings of the Strasbourg, Conference on quark-hadron phase transition.

Spite, F., Maillard, J.P., Spite., M., 1984, Astron. Astrophys. 141, 56.

Spite, F., Spite, M., 1982, Astron. Astrophys. 115, 357.

Spite, F., Spite, M., Peterson, R.C., Chafee, F.H., 1987, Astron. Astrophys. 172, L9.

Terasawa, N., Sato, K., 1989, Prog. Theor. Phys. Lett. 81, 254.

Terasawa, N., Sato, K., 1989, Phys. Rev. D 39, 2893.

Ukawa, A, 1988, Status of lattice QCD at finite temperature, CERN -TH 5266.

Vauclair, S., 1988, Astrophys. J. 335, 971.

Vangioni-Flamm, E., Audouze, J., 1988, Astron. Astrophys. 193, 81.

Witten, E., 1984, Phys. Rev. D30, 272.

Yang, J., Tumer, M.S., Steigman, G., Schramm, D.N., Olive, K., 1984, Astrophys. J. 281, 493. 